\title{
Channel Allocation Policy for Distributed Wireless Network: Derivation and Analysis of Optimal Interference
}

\author{
Amulya Bhattarai $(\mathbb{D}$, Prapun Suksompong $(\mathbb{D}$, and Chalie Charoenlarpnopparut \\ School of Information, Computer and Communication Technology (ICT), SIIT, Thammasat University, \\ Pathum Thani 12000, Thailand \\ Correspondence should be addressed to Prapun Suksompong; prapun@siit.tu.ac.th
}

Received 17 April 2020; Revised 5 October 2020; Accepted 10 October 2020; Published 6 November 2020

Academic Editor: Pietro Manzoni

Copyright (C) 2020 Amulya Bhattarai et al. This is an open access article distributed under the Creative Commons Attribution License, which permits unrestricted use, distribution, and reproduction in any medium, provided the original work is properly cited.

\begin{abstract}
Distributed wireless networks with smart users (independent and rational) are becoming popular, and researchers are studying distributed equilibrium solutions like Nash Equilibrium (NE) to analyze and predict the convergence of such networks. Our goal is to drive the distributed wireless network to NE with high total throughput. Study of the distribution of network metrics at NE with high total throughput shows that communication links still have significant amount of interference. Adding an interference-received term with an optimal weight $\left(\alpha_{\mathrm{opt}}^{*}\right)$ to the link's payoff can push the distributed network to converge to NE with high total throughput. The channel allocation trend at NE with high total throughput is as follows: each of the $C-1$ links occupies its own channel, and the remaining $N-C+1$ links share the remaining one channel, where $N$ is the number of links and $C$ is the number of channels in the network. The links (transmitters and receivers) are randomly located and $C<N$ (limited resources). The transmitter of a link has a direct connection with the receiver of the link; hence, several links overlap. This leads to a dense network with considerable amount of interference especially for links sharing channels. A practical application of our work is when smart devices in a room, hall, or concert arena have a direct communication with other smart devices in the area using limited bandwidth. Using best response technique and definitions of NE, we derive and propose an approximate way to mathematically express $\alpha_{\text {opt }}^{*}$ (referred to as $\widehat{\alpha}_{\text {opt }}$ ) along with its probability density function (PDF) for a specific scenario. Then, a generic equation for $\widehat{\alpha}_{\text {opt }}$ is inferred for varying network sizes (links) and available resources (channels). Implementing such a policy enhances the total throughput of the distributed wireless network by up to $15 \%$. In a more general setting, our distributed policy can achieve up to $75 \%$ of the maximum total throughput (benchmark value reached by centralized solution via exhaustive search) at a fraction of the time and computation resources.
\end{abstract}

\section{Introduction}

Wireless communication and networking is expanding exponentially [1]. Scientists are exploring new techniques to keep up with the escalated traffic. Various technologies in mobile communication (3G, 4G, and 5G) [2], wireless LAN (WiFi) [3], and personal area networking (Bluetooth) [4] are catering to the need of the present wireless communications, but new innovations and techniques are essential to meet up with the future trends and ever growing demand [5].

Fixed Resource Allocation (FRA) of power, spectrum, and transmission protocols are inefficient [6] as only a fraction of the allocated resources is utilized by the designated users. By dynamically allocating the resources, the smart radios can enhance the efficiency of the network by several folds in comparison to FRA [7]. Intelligent radios are capable to sense the environment, reason based on the observations made [8], and adopt their transmission parameters to get higher efficiency and performance [9]. In this work, we focus on dynamically allocating the channels (spectrum) to the intelligent wireless users [10].

As radios are becoming more intelligent and have greater computational power, distributed networks are becoming popular [11]. Several, mobile devices may connect and share information with each other via Bluetooth or WiFi in the absence of a central entity [4]. Generally, in a distributed 
network, each user independently makes the decision [12], i.e., each user acts selfishly to maximize its own payoff [13]. Although a central agent might ordain a global optimal strategy to these users, they will mostly drift away by choosing strategies that enhance their own payoff, consequently resulting in a lower overall network performance compared to the global optimal [14]. Centralized solutions via exhaustive search technique result in maximum total throughput; however, it requires massive amount of resources (computation and time) especially in larger networks. Hence, many networks like ad hoc and device to device (D2D) prefer decentralized solutions [15].

Nash Equilibrium (NE) is a natural and eventual equilibrium state, which the independent and selfish users converge to in distributed scenarios [16]. As each user tries to enhance its own payoff in every iteration, after considerable amount of iterations, the system converges to a Nash Equilibrium (NE). Depending on the starting point, the definition of a user's payoff, network size, resources available, etc., the system can converge to NE with varying performance [17]. We want to converge to a NE with high total throughput [18].

Several research groups have used game theory techniques to address the resource allocation problem in wireless distributed networks. The authors in [17] have defined the user's payoff to be the individual throughput. This is a distributed system and there is no information exchange between the users, but mostly, the system converges to NE solutions with poor performance [19]. In [20] the authors have defined the user's payoff to be the total throughput of all users to emulate a centralized system where each user works to maximize the total performance of the network. The performance is high; however, each user needs to know the throughput of all other users which is not viable for a practical distributed network. In [21], the authors have defined the user's payoff to be the received power minus interference received plus interference created to other users. The objective is to increase the received power and to minimize the interference received from other users and the interference a user generates to other users [22]. Here, the resource allocation schemes are performed in a distributed way, but complete information exchange among the users is essential, and the performance is only slightly better than its predecessors.

In $[23,24]$, the characteristics of the network metrics, link distance, individual throughput, and interference from/to, were studied. It was found that adding an optimal amount of interference-received term in the user's utility, the distributed wireless network can be driven towards NE with high total throughput [18]. There, $\alpha_{\mathrm{opt}}^{*}$ is the optimal weight of the interference-received term for a specific network which is determined by running many random simulations for step size variations of $\alpha$. In this work, we mathematically derive an approximate expression of $\alpha_{\mathrm{opt}}^{*}$ (referred to as $\widehat{\alpha}_{\mathrm{opt}}$ ) along with its probability density function (PDF), for a random "10-link 4-channel" scenario. Then, a generic equation of $\widehat{\alpha}_{\text {opt }}$ for an "N-link C-channel" scenario is inferred based on the trend of different network sizes. In our work, the links (transmitters and receivers) are randomly located and $C<N$ (limited resources). The transmitter of a link has a direct connection with the receiver of the link; hence, several links overlap. This leads to a dense network with considerable amount of interference especially for links sharing channels. A practical application of our work is when smart devices in a room, hall, or concert arena have a direct communication with other smart devices in the area using limited bandwidth. Implementing the policy enhances the total throughput of the distributed wireless network by up to $15 \%$, and it can achieve up to $75 \%$ of the maximum total throughput (the benchmark value reached by the centralized solution via exhaustive search technique) at a fraction of time and computational resources.

In Section 2, the system model of our distributed wireless network is explained. In Section 3, game theory is analyzed in detail. NE with high total throughput is defined, and how distributed solutions with high total throughput can be achieved by inserting the optimal weight $\left(\alpha_{\mathrm{opt}}^{*}\right)$ of the interferencereceived term in the utility is explained. Section 4 mathematically derives the policy $\left(\widehat{\alpha}_{\text {opt }}\right)$ from NE with high total throughput constraints for a "10-link 4-channel" scenario and extends to incorporate a generic solution for varying network sizes and available resources. Section 5 compares the results from centralized and distributed networks. Using various illustrations, it is shown how implementing our policy enhances the performance of the distributed wireless network. Section 6 concludes the work.

\section{System Model}

This section discusses the main components of the model. Initially, the links (pairs of receivers and transmitters) and channels in our model are described. Then, calculation of the received power, interference, and throughput at the links and network is explained.

2.1. Links and Channels. In our wireless network, there are $N$ links. Each link comprises of a transmitter $(\mathrm{Tx})$ and a receiver $(\mathrm{Rx})$. There are $i \in 1,2, \cdots, N$ transmitters and $j \in 1,2, \cdots, N$ receivers. Link $k_{j}$ comprises a transmitter $\mathrm{Tx}_{i}$ which has a direct connection with a receiver $\mathrm{Rx}_{j}$ where $i=j$. We assume that the maximum communication distance of $\mathrm{Link}_{j}$ is the entire network area. For example, Link $_{1}$ is the communication between $\mathrm{Tx}_{1}$ and $\mathrm{Rx}_{1}$. Figure 1 (a) shows a network with 10 links $(N=10)$ and 4 channels $(C=4)$. Symbols " *" and "o" represent the $\mathrm{Tx}_{i}$ and $\mathrm{Rx}_{j}$, respectively. We can observe that several links overlap creating a dense network with considerable amount of interference especially for links sharing channels. The coordinates of the $\mathrm{Tx}_{i}$ and $\mathrm{Rx}_{j}$ are randomly and uniformly generated inside an area of 10 units by 10 units.

Limited resources are available in the wireless network, and hence, channels need to be shared by the links. The resources allocated to $N$ links are $C$ channels. An interesting case is when there are less channels than the number of links: $C<N$. A practical application of our work is when smart devices in a room, hall, or concert arena have direct communication with other smart devices in the area using limited 


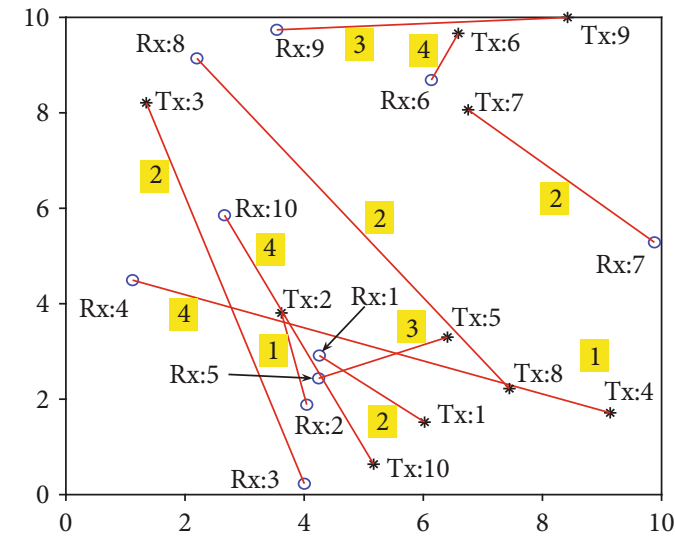

(a) There are a total of 10 links which share 4 channels ( $\operatorname{Link}_{1}$ is the communication between $\mathrm{Tx}_{1}$ and $\mathrm{Rx}_{1}$ which is using channel " 2 "). The coordinates of the $\mathrm{Tx}_{i}$ and $\mathrm{Rx}_{j}$ are randomly and uniformly generated

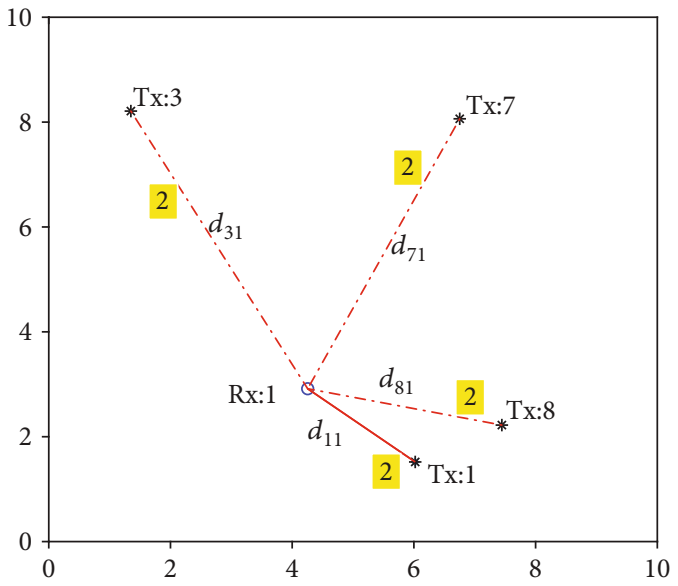

(b) The SINR of Link $k_{1}$ is the ratio of signal power (from $\operatorname{Tx}_{1}$ equaling $1 / d_{11}^{2}$ ) over the interference power (from $\mathrm{Tx}_{3}, \mathrm{Tx}_{7}$, and $\mathrm{Tx}_{8}$ equaling $1 / d_{31}^{2}+1 / d_{71}^{2}$ $\left.+1 / d_{81}^{2}\right)$ and noise power. These links use channel " 2 "

Figure 1: Distributed wireless network: random, "10-link 4-channel” scenario. The 10 links use a random channel configuration (initially) of “2124342234" (Link, Link $_{3}$, Link $_{7}$, and Link 8 use channel "2").

bandwidth. We assume each link can occupy only one channel at a time.

2.2. Mathematical Formulations. The power at the receiver from a transmitter, using the free space ideal propagation model, can be expressed as

$$
P_{r}=\frac{G_{r} G_{t} P_{t}}{(4 \pi d / \lambda)^{2}}=\frac{A}{d^{2}}
$$

where $G_{r}$ and $G_{t}$ are the antenna gain at the receiving and transmitting sides, respectively. $P_{r}$ and $P_{t}$ are the power at the receiver and transmitter, respectively. The distance between the transmitter and the receiver is $d$, and $\lambda$ is the wavelength of the electromagnetic wave used in the communication system [25]. In our work, the transmission power of all antennas and the gain of the antennas are constant. Similarly, as we are operating in a narrowband spectrum, we consider the wavelength of the electromagnetic wave used in the wireless network to be constant. These constants are combined into one constant $A$. Since the main idea is to explore the received power with the variation of the link distance, without loss of generality, we normalize $A$ to unity. The received power at $\mathrm{Rx}_{j}$ from $\mathrm{Tx}_{i}$ can then be simplified to

$$
p_{i j}=\frac{1}{d_{i j}^{2}},
$$

where $d_{i j}$ is the distance between $\mathrm{Tx}_{i}$ and $\mathrm{Rx}_{j}$. In order to avoid very high or infinite values of $p_{i j}$ when the distance is very small, we consider the minimum value of $d_{i j}$ to be 1 [26], avoiding near-field effects [27].
Then, the Signal to Interference plus Noise Ratio (SINR) of $\operatorname{Link}_{j}$ is

$$
\operatorname{SINR}_{j}=\frac{p_{i j}}{\sum_{j=1}^{N} p_{i j}+\sigma_{n}^{2}}=\frac{p_{i j}}{I_{j}^{\text {from }}+\sigma_{n}^{2}} \text { where } \quad \begin{gathered}
i=j \text {, same link, } \\
i \neq j \text {, different links. }
\end{gathered}
$$

In Equation (3), $\sigma_{n}^{2}$ is the noise power and $\sum_{j=1}^{N} p_{i j}$ is the total interference received from other links to Link ${ }_{j}$ denoted as $I_{j}^{\text {from }}$. The equation assumes all links share the same channel; however, only a certain number of links share a channel and there is interference only between links sharing the same channel as illustrated in Figure 1(b). SINR 1 equals the ratio of the power of the signal (due to the $\mathrm{Tx}_{1}$ which equals to $p_{11}$ $=1 / d_{11}^{2}$ ) over the power of the interference and noise. The random channel configuration (initially) used in Figure 1 is "2124342234" (first number signifying the channel used by Link 1 and henceforth). Link , Link $_{3}$, Link channel "2." Hence, for Link 1 , there is interference from T $\mathrm{x}_{3}, \mathrm{Tx}_{7}$, and $\mathrm{Tx}_{8}$ which equals to $I_{1}^{\text {from }}=1 / d_{31}^{2}+1 / d_{71}^{2}+1 /$ $d_{81}^{2}$. In this work, to make a concrete example, noise power $\left(\sigma_{n}^{2}\right)$ is arbitrarily set at 0.001 , which is $30 \mathrm{~dB}$ smaller than the transmit power.

The throughput of Link ${ }_{j}$ based on Shannon Capacity [28] with AWGN channel and SINR $_{j}$ [29] is expressed as

$$
T_{j}=\log _{2}\left(1+\operatorname{SINR}_{j}\right) .
$$

Here, the throughput is in bit $/ \mathrm{s} / \mathrm{Hz}$, i.e., the throughput is normalized over the bandwidth. 
The total throughput of a wireless network with $N$ links is the sum of all the throughput of $N$ links,

$$
T_{\text {tot }}=\sum_{j=1}^{N} T_{j}
$$

$T_{\text {tot }}$ varies as different links choose different strategies which will be further explained in Section 3. We define, $T_{\text {tot }}^{\max }$ to be the maximum $T_{\text {tot }}$ from all the different possible pure strategies obtained by exhaustive search of a random scenario.

2.3. Assumptions. Four notable assumptions have been made in this work, while focusing on distributed wireless networks, which are as follows:

First, the links compute the resource allocation schemes themselves in a distributed way and choose the strategies independently.

Second, the links are rational and selfish. Links will choose the strategies in order to increase their own payoff. In our work, utility is defined as in Equation (7) signifying a noncooperative network with independent and selfish users.

Third, there is no information sharing between links. Each link can measure the received power and interference it receives from other links assuming the number of links and channels available in the network are broadcasted in the beacon signal, like in cellular or WiFi networks [30].

Fourth, good error control techniques are implemented in the links. A system model as mentioned in Section 2.1 leads to a dense network with a significant amount of interference. In particular, links sharing channels face considerable amount of interference. We assume the links have good error control techniques which allow them to communicate with some small bit rate even in the presence high interference (as per Shannon's formula, Equation (4)). Over iterations, links could find other channels that have lower interference and decide to transmit at increased data rate as well.

Based on the system model and assumptions stated above, channel allocation policy in our distributed wireless network will be analyzed using game theory in the next section.

\section{Game Formulation}

Game theory is a mathematical tool that is used to analyze the strategic interaction of players, especially with conflicting interests. In recent years, game theory has been used in distributed wireless networks for resource allocation purposes.

A game can be symbolically expressed as a triplet, $<N$, $S_{j}, U_{j}\left(S_{j}, S_{-j}\right)>$ such that there are $N$ players (links) in the game, $j \in 1,2, \cdots, N$. $S_{j}$ is the set of strategies of player $j$, and $S_{-j}$ represents the strategies played by all players apart from $j$. The utility or payoff of player $j\left(\operatorname{Link}_{j}\right)$ is $U_{j}$, which is a function of the strategy chosen by all players [16]. In this work, the players are the links. Each link chooses to transmit in a particular channel resulting in a different payoff. Rational and independent users will try to maximize their $U_{j}$ in every iteration [31]. After certain iterations, they mostly converge to a Nash Equilibrium (NE) [32].

3.1. Nash Equilibrium (NE). Nash Equilibrium (NE) is a natural and eventual equilibrium state reached by rational and independent players in a distributed network. The players choose strategies that maximize their individual utility. At $\mathrm{NE}$, each player will not be able to increase its utility by unilaterally changing its strategy if the strategies of other players remain unchanged [16], which is expressed mathematically as

$$
U_{j}\left(S_{j}^{*}, S_{-j}^{*}\right) \geq U_{j}\left(S_{j}, S_{-j}^{*}\right) \quad \forall j \in 1,2, \cdots, N
$$

where $S_{j}^{*}$ represents the best response strategy of link $j$ and $S_{-j}^{*}$ represents the best response strategy of all other links except $j$.

A centralized global solution may result in a higher overall performance, but once the rational and selfish players are allowed to make their independent choices, they will drift away from the centralized global solution. However, once the system converges to a NE, none of the rational players will change their strategies as, by doing so, they will get a lower $U_{j}$ as indicated in Equation (6). Moreover, centralized solutions via an exhaustive search technique require a massive amount of resources (computational and time) especially in larger networks which is unfeasible. Hence, many practical systems prefer distributed solutions.

In the next section, we define the utility of a link such that the distributed system will converge to NE with higher performance.

3.2. Varying Utility to Converge to NE with High Performance. In $[18,24]$, total throughput of the network with varying links and channels was analyzed using an exhaustive search method. Distribution of network metrics $\left(T_{j}, I_{j}^{\text {from }}\right.$, and $\left.d_{i j}\right)$ reflect that links have a significant amount of interference at NE with high total throughput.

In order to converge to NE solution with high total throughput, the utility of a link in this work is defined as

$$
U_{j}=T_{j}+\alpha_{\mathrm{opt}}^{*} \cdot I_{j}^{\mathrm{from}}=\log _{2}\left(1+\frac{1 / d_{i j}^{2}}{I_{j}^{\mathrm{from}}+P_{n}}\right)+\alpha_{\mathrm{opt}}^{*} \cdot I_{j}^{\mathrm{from}},
$$

where $T_{j}$ is the individual link's throughput as defined in Equation (4), $I_{j}^{\text {from }}$ is the interference received from other links to Link ${ }_{j}$ as defined in Equation (3), $\alpha$ is the weight of $I_{j}^{\text {from }}$, and $d_{i j}$ is the distance between the transmitter and receiver of Link ${ }_{j}$.

Adding an optimal interference term to the payoff of a link might sound contradictory; however, such characteristics are observed in different spectra of life as well. For example, let us consider a city with a certain population having a limited number of vehicles. An equilibrium point with better well-being can be achieved when the vehicles are shared 
optimally [33]. By sharing the vehicles, the users have to experience inconveniences like longer ride time and route; however, the overall traffic congestion in the city can be reduced. Hence, the government can give benefits or other incentives to encourage the users in the city to share vehicles to enhance the well-being of the entire city. Similarly, when the wireless users in a distributed network voluntarily (or are hard-wired to) tolerate some level of interference in their payoff, the network can achieve solutions with higher performance. Another befitting example is of the tax payed by a citizen to the government of its country. No one really wants to pay taxes as its being cut off from the salary earned from hard work. Similarly, none of the links in a network wants to share the channel and the implicated interference, which thereby diminishes its throughput. However, all citizens have to pay taxes which are ideally used to run the government and provide social security/benefits like health care, education, and peace/security for the people of the country. Paying taxes facilitates the government to run better, so we can live in a better community [34]. Similarly, if each link in the network tolerates certain levels of interference, then the network can operate at a higher total throughput.

Figure 2 illustrates the convergence characteristics of the distributed system. The links use a best response technique to converge. In every iteration, each user chooses a strategy that maximizes its own utility, and eventually, the system converges to NE [32]. Generally, the best response technique converges to $\mathrm{NE}$ if the game is a potential function [35]. However, in our work, we start by assuming that the system converges to $\mathrm{NE}$ at a particular channel configuration (that results in high $T_{\text {tot }}$ ) as explained in Section 4.1. Then, from the NE constraints, we derive the PDF and mean value of $\alpha_{\text {opt }}$ which is the key parameter in the utility function (Equation (7)). So, using the best response technique in the derived utility pushes the distributed network to converge to NE (mostly) with high performance. In a general distributed system, each user tried to maximize its individual throughput, that is, $U_{j}=T_{j}$. However, the converged solution of the overall system is not so high. Adding an optimal amount of interference in the utility $\left(U_{j}=T_{j}+\alpha_{\text {opt }}^{*} \cdot I_{j}^{\text {from }}\right.$, Equation (7)) pushes the system to converge to NE with high $T_{\text {tot }}$. In Section 4 , we mathematically derive the approximate value of $\alpha_{\text {opt }}^{*}$ (referred to as $\widehat{\alpha}_{\text {opt }}$ ) which gives similar performance at a fraction of the time and computation resources. Figure 2 shows the average $T_{\text {norm.cum }}^{\text {tot }}$ (normalized cumulative total throughput [24]) from 1000 random scenarios in the $y$-axis and the number of iterations (time) in the $x$-axis.

In the upcoming section, we formulate the policy and derive an approximate mathematical expression and PDF for $\alpha_{\text {opt }}^{*}$ (referred to as $\widehat{\alpha}_{\text {opt }}$ ) from NE constraints that results in high $T_{\text {tot }}$ for a random "10-link 4-channel" scenario and for varying links and channels.

\section{Proposed Method for Approximating the Bounds and PDF of $\alpha_{\mathrm{opt}}^{*}$}

In this section, we are going to approximately derive some bounds and PDF of $\alpha_{\text {opt }}^{*}$ (referred hereafter as $\widehat{\alpha}_{\text {opt }}$ ) that need to be in the individual link's utility to converge to a NE solution with high $T_{\text {tot }}$ for a random "10-link 4-channel" scenario then for scenarios with a varying number of links and channels. $\widehat{\alpha}_{\text {opt }}$ is quite close to $\alpha_{\text {opt }}^{*}$, but the main advantage of $\widehat{\alpha}_{\text {opt }}$ is that it can be computed in a fraction of the time and computational resources required to compute $\alpha_{\mathrm{opt}}^{*}$.

To proceed with the analysis, shared utility is defined based on the definition of a link's utility expressed in Equation (7): when a link gets a single channel, the utility of the link is $U_{s 1}$ which equals to $\log _{2}\left(1+\left(1 / d_{a}^{2}\right) / P_{n}\right)$. There is no interference received from other links for $U_{s 1}$. When $l$ links share a channel, the utility of the link is defined as $U_{s l}$ which is expressed as

$U_{s l}=\log _{2}\left(1+\frac{1 / d_{a}^{2}}{\sum_{m=1}^{l-1}\left(1 / d_{\mathrm{Im}}^{2}\right)+P_{N}}\right)+\alpha_{\mathrm{opt}}^{*} \cdot\left(\sum_{m=1}^{l-1} \frac{1}{d_{\mathrm{Im}}^{2}}\right) \quad$ for $N \geq 2$

Here, $d_{a}$ is the distance of the signal between the transmitter and the receiver of the same link and $d_{\mathrm{Im}}$ is the distance from the $m^{\text {th }}$ interfering link where $m=1,2, \cdots, N-1$.

The probability density function (PDF) of $1 / d_{a}^{2}$ is illustrated in Figure 3. $d_{a}$ is the distance between the transmitter (with coordinates $x_{2}$ and $y_{2}$ ) and the receiver (with coordinates $x_{1}$ and $y_{1}$ ) and can be mathematically expressed as $\sqrt{\left(x_{2}-x_{1}\right)^{2}+\left(y_{2}-y_{1}\right)^{2}} \cdot x_{1}, x_{2}, y_{1}$, and $y_{2}$ are random variables with uniform distribution between 0 and 10 units as illustrated in Figure 1(a). High probability mass occurs at the maximum value of $1 / d_{a}^{2}=1$, as per our assumption in Equation (2). For simplicity of analysis, the distribution of the interfering links $d_{\operatorname{Im}}(m=1,2, \cdots, l-1)$ is assumed to have the same distribution as $d_{a}$. This distribution is used to compute the PDF of $\alpha_{\mathrm{opt}}^{*}$ in the upcoming section.

4.1. The "10-Link 4-Channel" Scenario. For "10 links 4 channels," it has been observed that the maximum $T_{\text {tot }}$ occurs mostly $(\approx 85 \%)$ at channel configuration "1234444444" [18]. The left portion of Figure 4 considers the first link $(j=1)$ which is getting a "single channel," and the right portion considers the fourth link $(j=4)$ which is "sharing channel" with other links. The first, second, and third links get a "single channel" and have a payoff of $U_{s 1}$, as defined in Equation (8). Once the strategy is changed, the payoff of the link will be either $U_{s 2}$ if channel 2 or 3 is selected or $U_{s 8}$ if channel 4 is selected. Hence, the NE conditions are $U_{s 1} \geq U_{s 2}$ and $U_{s 1}$ $\geq U_{s 8}$. The fourth to tenth links share channel 4, so the payoff is $U_{s 7}$. Once the strategy is changed to channel 4, the payoff of the link will be $U_{s 2}$. Hence, the NE condition is $U_{s 7} \geq U_{s 2}$.

Since the value of optimal $\alpha$ obtained from the condition $U_{s 1} \geq U_{s 2}$ is larger than the value obtained from the condition $U_{s 1} \geq U_{s 3}$ which is larger than the value obtained from the condition $U_{s 1} \geq U_{s 4}$ and so forth as illustrated in Figure 5, the upper bound for optimal $\alpha$ for the above mentioned conditions is exponentially decreasing. As $U_{s 1} \geq U_{s 2}$ is a subset of $U_{s 1} \geq U_{s 8}$, we get one unique upper bound 


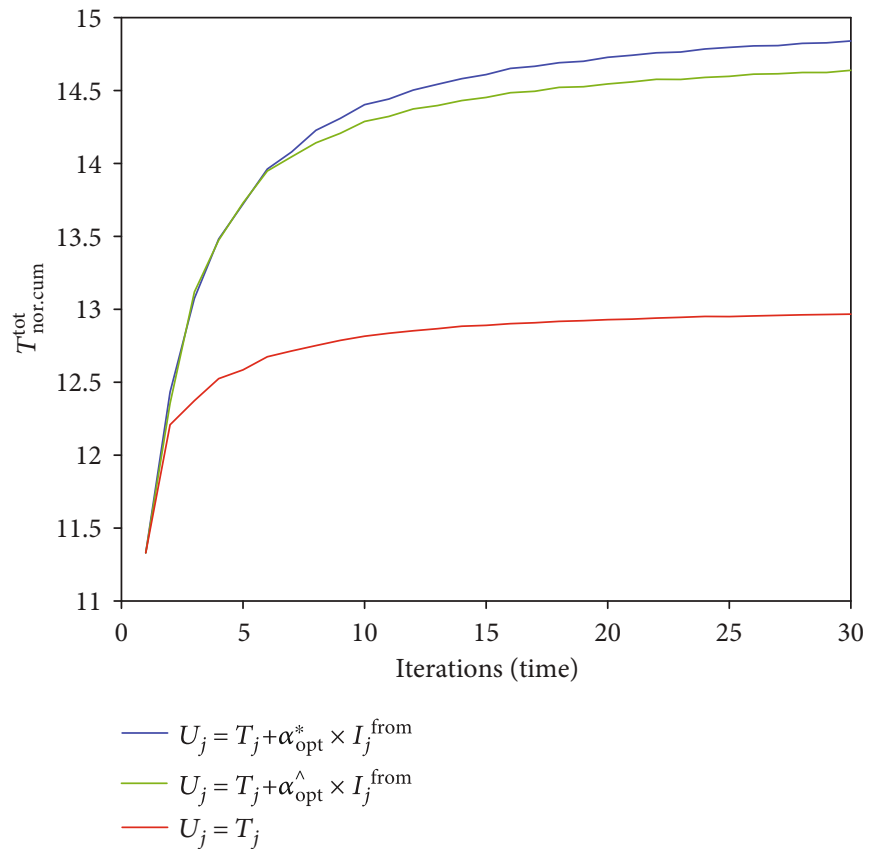

FIGURE 2: Convergence characteristics (iterations versus $T_{\text {nor.cum }}^{\text {tot }}$ ) of the distributed system for different definitions of utility. Best response technique is used to converge to NE. An average of 1000 random "10-link 4-channel" scenarios is considered.

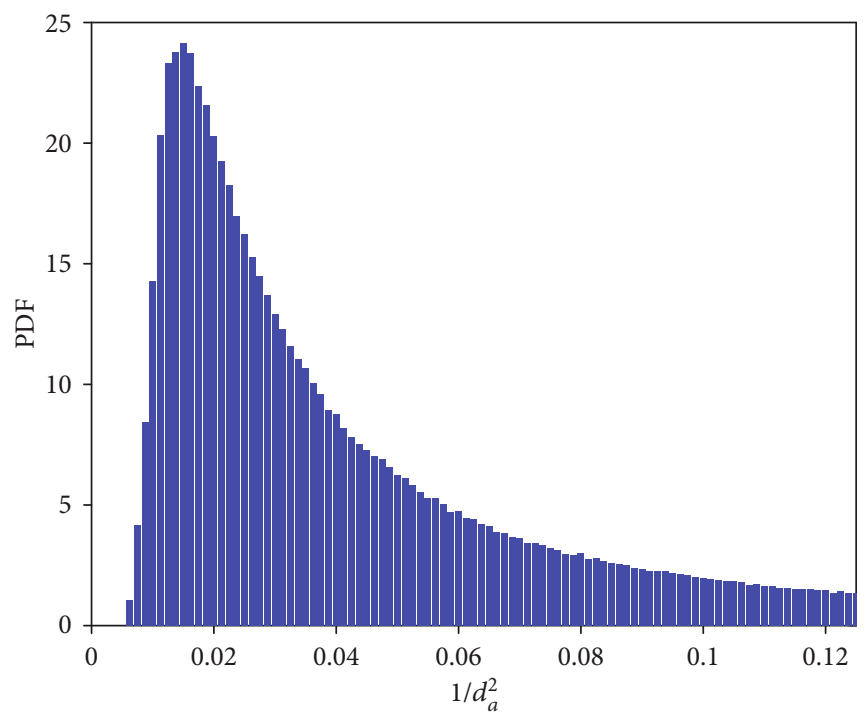

FIgURE 3: Probability density function (PDF) of $1 / d_{a}^{2} \cdot d_{a}$ is the distance of the signal between transmitter and receiver of the same link.

(UB) condition: $U_{s 1} \geq U_{s 8}$, and one lower bound (LB) condition: $U_{s 7} \geq U_{s 2}$.

$U B$ condition: solving for $U_{s 1} \geq U_{s 8}$, using the definition of shared utility from Equation (8) when $l=1$ and 8 results in

$\log _{2}\left(1+\frac{1 / d_{a}^{2}}{P_{N}}\right) \geq \log _{2}\left(1+\frac{1 / d_{a}^{2}}{1 / d_{I 1}^{2}+\cdots+1 / d_{I 7}^{2}+P_{N}}\right)+\alpha\left(\frac{1}{d_{I 1}^{2}}+\cdots+\frac{1}{d_{I 7}^{2}}\right)$,

$\alpha_{\mathrm{opt}}^{\mathrm{UB}} \leq \frac{\left[\log _{2}\left(1+\left(1 / d_{a}^{2}\right) / P_{N}\right)-\log _{2}\left(1+\left(1 / d_{a}^{2}\right) /\left(1 / d_{I 1}^{2}+\cdots+1 / d_{I 7}^{2}+P_{N}\right)\right)\right]}{\left(1 / d_{I 1}^{2}+\cdots+1 / d_{I 7}^{2}\right)}$.
$L B$ condition: solving for $U_{s 7} \geq U_{s 2}$, using the definition of shared utility from Equation (8) when $l=7$ and 2 results in

$$
\begin{gathered}
\log _{2}\left(1+\frac{1 / d_{a}^{2}}{1 / d_{I 1}^{2}+\cdots+1 / d_{I 6}^{2}+P_{N}}\right)+\alpha\left(\frac{1}{d_{I 1}^{2}}+\cdots+\frac{1}{d_{I 6}^{2}}\right) \geq \log _{2}\left(1+\frac{1 / d_{a}^{2}}{1 / d_{I 1}^{2}+P_{N}}\right)+\alpha\left(\frac{1}{d_{I 1}^{2}}\right), \\
\alpha_{o p t}^{L B} \geq \frac{\left[\log _{2}\left(1+\left(1 / d_{a}^{2}\right) /\left(1 / d_{I 1}^{2}+P_{N}\right)\right)-\log _{2}\left(1+\left(1 / d_{a}^{2}\right) /\left(1 / d_{I 1}^{2}+\cdots+1 / d_{I 6}^{2}+P_{N}\right)\right)\right]}{\left(1 / d_{I 2}^{2}+\cdots+1 / d_{I 6}^{2}\right)} .
\end{gathered}
$$

$\alpha_{\mathrm{opt}}^{\mathrm{UB}}$ is larger than $\alpha_{\mathrm{opt}}^{\mathrm{LB}}$ because the first term of $\alpha_{\mathrm{opt}}^{\mathrm{UB}}$ is much larger than the first term of $\alpha_{\mathrm{opt}}^{\mathrm{LB}}$. The absence of $1 / d_{I 1}^{2}$ 


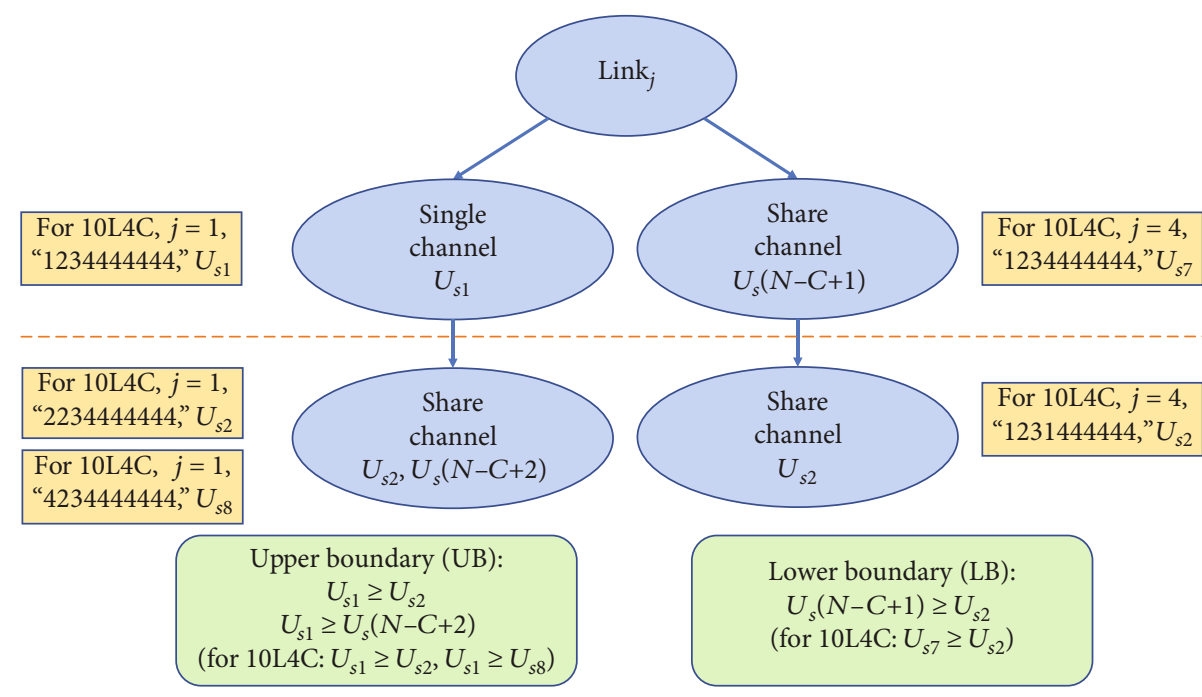

Figure 4: At NE with high $T_{\text {tot }}$, Link ${ }_{j}$ can use a "single channel" or "share channel" with other links. Based on the channel configuration at $\mathrm{NE}_{\text {Good }}$, the possible outcome after changing the strategy can be categorized. Applying the definition of NE, we can come up with the upper bound (UB) and lower bound (LB) conditions of optimal $\alpha$.

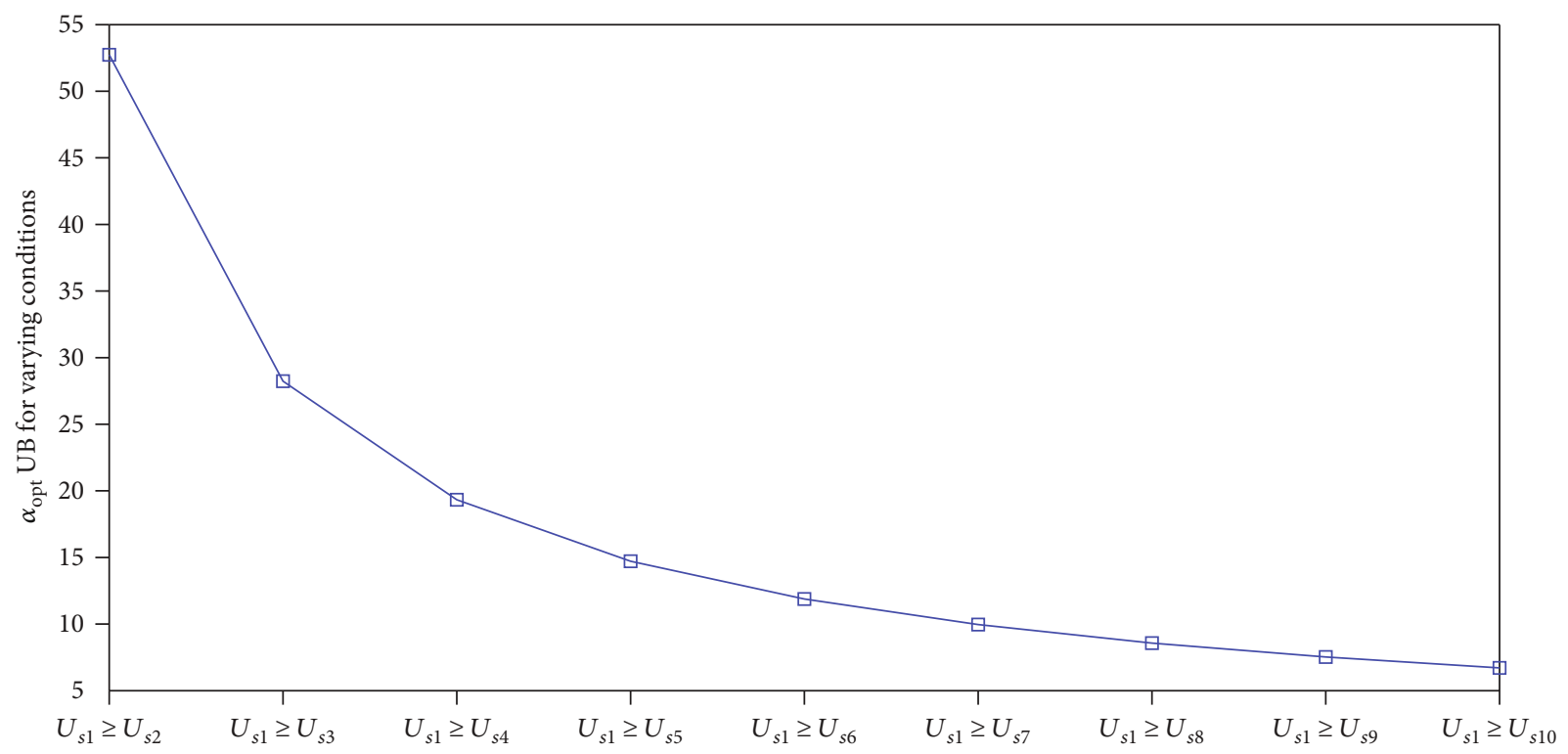

FIGURE 5: Upper bound (UB) of optimal $\alpha$ for varying conditions.

interfering expression (which is much larger than $P_{N}$ ) in the denominator of the first term of $\alpha_{\mathrm{opt}}^{\mathrm{UB}}$ produces such a result, $\left[\log _{2}\left(1+\left(1 / d_{a}^{2}\right) / P_{N}\right) \gg \log _{2}\left(1+\left(1 / d_{a}^{2}\right) /\left(1 / d_{I 1}^{2}+P_{N}\right)\right)\right]$. This holds true for the " $N$-link $C$-channel" scenario presented in the next section as well. $\alpha_{\mathrm{opt}}^{\mathrm{UB}}$ and $\alpha_{\mathrm{opt}}^{\mathrm{LB}}$ are computed using the PDF of $1 / d_{a}^{2}$ and $1 / d_{\mathrm{Im}}^{2}$. For a random "10-link 4channel" scenario, the PDF of $\widehat{\alpha}_{\text {opt }}$ is computed by taking the average of $\alpha_{\mathrm{opt}}^{\mathrm{UB}}$ and $\alpha_{\mathrm{opt}}^{\mathrm{LB}}$, which is illustrated in Figure 6 and has a mean value of 7.03 units. $\alpha_{\mathrm{opt}}^{*}$ for this network is determined as such: the value of $\alpha$ is stepwise varied from 0 to 30 with an increment of 0.1 . For each value of $\alpha$, many random simulations are run and the average value of $T_{\text {tot }}$ is stored. The $\alpha$ value that results in maximum $T_{\text {tot }}$ is defined as $\alpha_{\mathrm{opt}}^{*}$ [18]. For this network $\alpha_{\mathrm{opt}}^{*}$ is equal to 6.8 units which is close to the value of $\widehat{\alpha}_{\text {opt }}$. In the next section, we are going to generalize the mathematical expression of $\widehat{\alpha}_{\text {opt }}$ for varying network sizes.

4.2. The "N-Link C-Channel" Scenario. In this section, we are going to deduce an expression of $\widehat{\alpha}_{\text {opt }}$ for different network sizes, with varying numbers of links $(N)$ and channels $(C)$.

Generalizing the findings from the the Section 4.1, NE with high $T_{\text {tot }}$ mostly occurs when $(C-1)$ links get a single channel each and the remaining channel is shared by $(N-C+1)$ links. As illustrated in Figure 4, Link $_{j}$ can be sharing a channel or using it alone at $\mathrm{NE}$ with high $T_{\text {tot }}$ and 


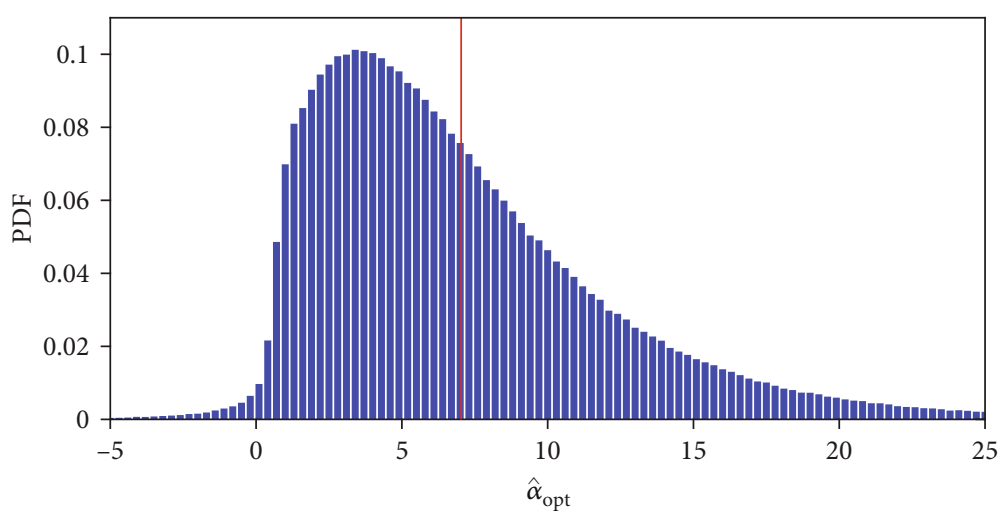

FIGURE 6: Probability density function (PDF) of $\widehat{\alpha}_{\text {opt }}$ for "10-link 4-channel" scenario; the mean is 7.03 units.

changing its strategy should yield in lower utility. The left portion of the flow chart shows that initially Link ${ }_{j}$ is using the strategy "single channel." Its payoff is $U_{s 1}$ as it is not sharing its channel with any other link. When the link changes its strategy to "share channel," it results in having two different payoffs. First, it will have to share a channel with a link which was previously getting a single channel, then its payoff will be $U_{s 2}$. Secondly, it will share a channel with links that were previously sharing a channel with $(N-C+1)$ links, then its new payoff will be $U_{s(\mathrm{~N}-\mathrm{C}+2)}$. Hence, the UB condition to achieve NE with high $T_{\text {tot }}$ are $U_{s 1} \geq U_{s 2}$ and $U_{s 1} \geq U_{s(N-C+2)}$. The first condition is the subset of the second condition which was explained earlier and illustrated in Figure 5. The right portion of the flow chart shows that initially, Link $\mathrm{j}_{j}$ is using the strategy "shared channel." Its payoff is $U_{s(N-C+1)}$ as it is sharing its channel with $(N-C+1)$ links. When the link changes the strategy, it will share a channel with the link which was previously using a "single channel." So, its new payoff will be $U_{s 2}$ . Hence, the LB condition is $U_{s(N-C+1)} \geq U_{s 2}$.

An expression to compute the expected value of $\widehat{\alpha}_{\text {opt }}$ for the " $N$-link $C$-channel" scenario can be computed by taking an expected average of $\alpha_{\mathrm{opt}}^{\mathrm{UB}}$ and $\alpha_{\mathrm{opt}}^{\mathrm{LB}}$ as follows:

$\widehat{\alpha}_{\text {opt }}^{-}=\frac{\left[\log _{2}\left(1+\left(1 / d_{a}^{2}\right) / P_{N}\right)-\log _{2}\left(1+\left(1 / d_{a}^{2}\right) /\left(\sum_{n=1}^{N-C+1}\left(1 / d_{\mathrm{In}}^{2}\right)+P_{N}\right)\right)\right] / \sum_{n=1}^{N-C+1}\left(1 / d_{\mathrm{In}}^{2}\right)+\left[\log _{2}\left(1+\left(1 / d_{a}^{2}\right) /\left(1 / d_{I 1}^{2}+P_{N}\right)\right)-\log _{2}\left(1+\left(1 / d_{a}^{2}\right) /\left(\sum_{n=1}^{N-C}\left(1 / d_{\mathrm{In}}^{2}\right)+P_{N}\right)\right)\right] / \sum_{n=1}^{N-C-1}\left(1 / d_{\mathrm{In}}^{2}\right)}{2}$

Equation (11) holds true for varying values of $N$ and $C$, but better results are obtained when $N / C \geq 1.8$. In the next section, we will compare the results and analyze the performance while implementing our policy: $\alpha_{\mathrm{opt}}^{*}$ and its approximate mathematical expression $\widehat{\alpha}_{\text {opt }}$ along with benchmark schemes.

\section{Results: Comparing Centralized and Distributed (Basic, $\alpha_{\mathrm{opt}}^{*}$, and $\widehat{\alpha}_{\mathrm{opt}}$ ) Schemes}

In this section, we are going to compare the results obtained by implementing our policy with a standard centralized and basic distributed network. Figure 7 plots the $T_{\text {tot }}$ for four different schemes in the $y$-axis and varying network sizes in the $x$-axis. The maximum value of $T_{\text {tot }}$ for each scenario is obtained from the centralized solution via an exhaustive search technique. In the exhaustive search method, all possible channel configurations are listed and $T_{\text {tot }}$ is sorted to obtain $T_{\text {tot }}^{\max }$. This is denoted by the blue line. For large networks, it is not possible to perform the exhaustive search method. For example, for the "13-link 6-channel" scenario, the total number of channel configurations is $6^{13}$ which is over 13 billion. So, the exhaustive search results for "13L6C" and larger scenarios is omitted. The lowest $T_{\text {tot }}$ is obtained when $U_{j}=T_{j}$, which represents a basic distributed wireless network (gray line). The value of $T_{\text {tot }}$ obtained by implementing the policy $U_{j}=T_{j}+\alpha_{\mathrm{opt}}^{*} \times I_{\text {from }}$ is illustrated by the red line. $\alpha_{\mathrm{opt}}^{*}$ is the optimal value of $\alpha$, which is obtained by varying the value of $\alpha$ for a wide range (here, from $0: 0.2: 50$ ) and choosing the value of $\alpha$ that results in maximum $T_{\text {tot }}$. In this work, we proposed a mathematical expression that approximates the real optimal $\alpha$ which is referred to as $\widehat{\alpha}_{\text {opt }}$, represented by the orange line. Computing the optimal $\alpha$ via the proposed approximation is much faster and consumes only a fraction of computational resources, and the performance is quite close to $\alpha_{\mathrm{opt}}^{*}$, as illustrated in Table 1. Implementing the policy can enhance the total throughput of a distributed network by up to $15 \%$ as illustrated in Figure 7.

Table 1 enlists seven different network sizes with varying links $(N)$ and channels $(C)$. As $N$ and $C$ of a network increases, the total possible channel configurations drastically increase to $C^{N}$, which is shown in the second column of the table. The 3rd column lists the time consumed to run 


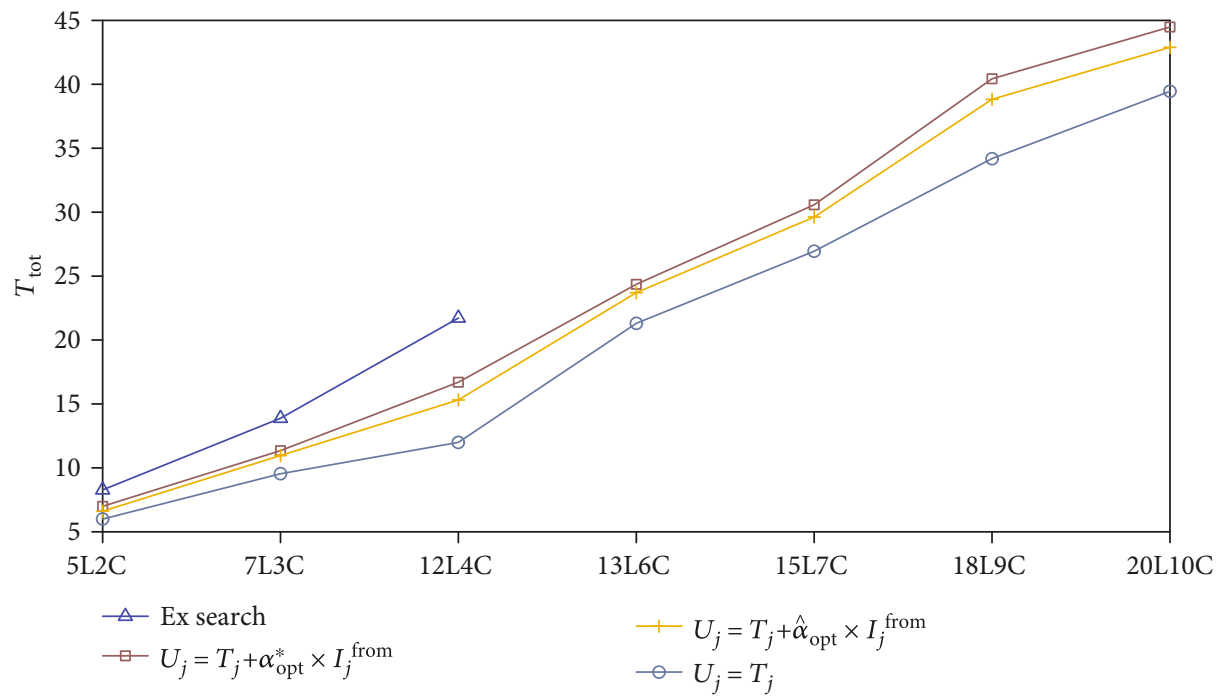

FIGURE 7: Illustration of how the distributed policy (adding $\widehat{\alpha}_{\text {opt }} \times I_{\text {from }}$ term in the utility) increases the $T_{\text {tot }}$ by up to $15 \%$ for distributed wireless networks. $\alpha_{\text {opt }}^{*}$ is the value of optimal $\alpha$ obtained from many random simulations for a wide range of $\alpha$. $\widehat{\alpha}_{\text {opt }}$ is obtained from a mathematical expressions that approximates the $\alpha_{\mathrm{opt}}^{*}$ and can be obtained at a fraction of computational and time resources. Although, the centralized solution from exhaustive search is the ideal solution, it is not feasible to adopt it especially for larger networks, due to its tremendous consumption of time and computational resources.

TABLE 1: Comparing the resources (time and computation) needed and the performance of the converged solution by centralized scheme via exhaustive search technique and our distributed policy (adding $\widehat{\alpha}_{\text {opt }} \times I_{\text {from }}$ term in the utility).

\begin{tabular}{lccc}
\hline Network & \multicolumn{2}{c}{$\begin{array}{c}\text { Centralized solution via exhaustive search } \\
\text { Time }(\text { estimated) consumed }\end{array}$} & $\begin{array}{c}\text { Distributed solution, policy }\left(\widehat{\alpha}_{\text {opt }}\right) \\
\text { Time consumed }\end{array}$ \\
\hline 5L2C & $2^{5}=3.2 \times 10^{1}$ & $32 \tau$ milliseconds & $39 \tau \mathrm{ms}$ \\
7L3C & $3^{7} \approx 2.19 \times 10^{3}$ & $8.74 \tau$ seconds & $42 \tau \mathrm{ms}$ \\
12L4C & $4^{12} \approx 1.68 \times 10^{7}$ & $18.64 \tau$ hours & $48 \tau \mathrm{ms}$ \\
13L6C & $6^{13} \approx 1.31 \times 10^{10}$ & $20 \tau$ months & $50 \tau \mathrm{ms}$ \\
15L7C & $7^{15} \approx 4.75 \times 10^{12}$ & $602 \tau$ years & $53 \tau \mathrm{ms}$ \\
18L9C & $9^{18} \approx 1.50 \times 10^{17}$ & $1.90 \times 10^{7} \tau$ years & $56 \tau \mathrm{ms}$ \\
20L10C & $10^{20} \approx 1.00 \times 10^{20}$ & $1.27 \times 10^{10} \tau$ years & $60 \tau \mathrm{ms}$ \\
\hline
\end{tabular}

all the $C^{N}$ channel configurations and find $T_{\text {tot }}^{\max }$. Computation time for single configuration is around $\tau=4 \mathrm{~ms}$ (considered as a unit time) in our desktop computer. The fourth column shows the time consumed (computing $\widehat{\alpha}_{\text {opt }}$ plus running best response technique) using the distributed policy proposed in this work. From the table, it is evident that as the link size increases, it is impossible (due to time and computational resources required) to go through each channel configuration and obtain $T_{\text {tot }}^{\max }$. Implementing the policy, the system can reach to a distributed NE solution with $\approx 75 \%$ of the $T_{\text {tot }}^{\max }$ consuming a fraction of the time and computational resources in comparison to the general distributed system $\left(U_{i}=T_{i}\right)$.

\section{Conclusion}

With the increment of smart users (independent and rational), distributed and decentralized wireless networks are gaining prominence. In such networks, there is no central entity that supervises/decides the strategies of each user, and the users do not share information with each other as well. Our network has many links (with a direct communication between a transmitter and receivers) within a limited area. Hence, links generally overlap, and when resources are limited $(C<N)$, there is high interference especially to links sharing channels. If the communication distance is limited to a certain range and channels are spatially reused, a different solution is expected (we plan to explore it further in our future work). The smart user in these networks tries to maximize its own utility in every iteration, and eventually, the network converges to an equilibrium solution called the Nash Equilibrium (NE). However, not all NE have high performance. From the analysis of different network metrics, it was found that communication links still have significant amount of interference values at $\mathrm{NE}$ with high $T_{\text {tot }}$. Adding 
weighted interference-received term in the payoff of a link can drive the decentralized system to converge to a solution with higher $T_{\text {tot }}$. In this work, we derived an approximate mathematical expression along with the PDF for $\alpha_{\mathrm{opt}}^{*}$, referred as $\widehat{\alpha}_{\text {opt }}$ for a random "10-link 4-channel" scenario. Then, we extracted the general trend of channel configuration at NE with high $T_{\text {tot }}$ and proposed a mathematical expression for $\widehat{\alpha}_{\text {opt }}$ for varying links $(N)$ and channels $(C)$.

Some limitations of our work are the value of noise power, area/dimension of the network, and the wireless path loss exponent is fixed. In reality, these values can vary which may result in different channel allocation at NE with high total throughput. This might cause a shift in the value of $\alpha_{\mathrm{opt}}^{*}$ and pdf of $\widehat{\alpha}_{\mathrm{opt}}$. When we derived a generic equation for $\widehat{\alpha}_{\text {opt }}$, we obtained the NE conditions from the channel allocation: "each of the $C-1$ links occupies its own channel and the remaining $N-C+1$ links share the remaining one channel" because, most of the time, NE with high $T_{\text {tot }}$ occurs at the channel configuration. However, at times especially when the ratio of $N / C$ is less than 1.8, NE with high $T_{\text {tot }}$ occurs at other channel allocations. We plan to address these issues in our future work.

In this work, we implement the policy and addition of an optimally weighted $\left(\widehat{\alpha}_{\text {opt }}\right)$ interference-received term in the payoff of a communication link. This enhances the performance of the distributed wireless network by up to $15 \%$, and the system can achieve up to $75 \%$ of the maximum total throughput (benchmark value reached by centralized solution via exhaustive search technique) at a fraction of the time and computation resources.

\section{Data Availability}

The data used to support the findings of this study are available from the corresponding author upon request.

\section{Conflicts of Interest}

The authors declare that they have no conflicts of interest.

\section{Acknowledgments}

The authors would like to thank Asst. Prof. Dr. Somsak Kittipiyakul, Dr. Attaphongse Taparugssanagorn, Assoc. Prof. Dr. Steven Gordon, and Assoc. Prof. Dr. Komwut Wipusitwarakun for their valuable suggestions and comments. This research is partially supported by the Excellent-ForeignStudents (EFS) scholarship from the Sirindhorn International Institute of Technology, Thammasat University.

\section{References}

[1] M. Agiwal, A. Roy, and N. Saxena, "Next generation 5G wireless networks: a comprehensive survey," IEEE Communications Surveys \& Tutorials, vol. 18, no. 3, pp. 1617-1655, 2016.

[2] S. Chen, J. Zhao, and Y. Peng, "The development of TDSCDMA 3G to TD-LTE-advanced 4G from 1998 to 2013," IEEE Wireless Communications, vol. 21, no. 6, pp. 167-176, 2014.
[3] J. G. Andrews, H. Claussen, M. Dohler, S. Rangan, and M. C. Reed, "Femtocells: past, present, and future," IEEE Journal on Selected Areas in Communications, vol. 30, no. 3, pp. 497508, 2012.

[4] T. Salonidis, P. Bhagwat, L. Tassiulas, and R. LaMaire, "Distributed topology construction of Bluetooth wireless personal area networks," IEEE Journal on Selected Areas in Communications, vol. 23, no. 3, pp. 633-643, 2005.

[5] H. Zhu, D. Niyato, W. Saad, and T. Basar, Game Theory for Next Generation Wireless and Communication Networks Modeling, Analysis and Design, Cambridge University Press, University of Cambridge, UK, 2019.

[6] FCC, "FCC reports, spectrum policy task force," 2002, http:// www.fcc.gov/sptf/reports.html.

[7] K. Zhu, D. Niyato, P. Wang, and Z. Han, "Dynamic Spectrum Leasing and Service Selection in Spectrum Secondary Market of Cognitive Radio Networks," IEEE Transactions on Wireless Communications, vol. 11, no. 3, pp. 1136-1145, 2012.

[8] J. Mitola and G. Q. Maguire, "Cognitive radio: making software radios more personal," IEEE Personal Communications, vol. 6, no. 4, pp. 13-18, 1999.

[9] S. Haykin, "Cognitive radio: brain-empowered wireless communications," IEEE Journal on Selected Areas in Communications, vol. 23, no. 2, pp. 201-220, 2005.

[10] R. Xie, H. Ji, P. Si, and Y. Li, "Dynamic channel and power allocation in cognitive radio networks supporting heterogeneous services," in 2010 IEEE Global Telecommunications Conference GLOBECOM 2010, pp. 1-5, Miami, FL, USA, December 2010.

[11] K. Moshksar and A. K. Khandani, "Decentralized wireless networks: spread spectrum communications revisited," IEEE Transactions on Information Theory, vol. 60, no. 5, pp. 25762593, 2014.

[12] Y. Zhang and C. Leung, "A distributed algorithm for resource allocation in OFDM cognitive radio systems," IEEE Transactions on Vehicular Technology, vol. 60, no. 2, pp. 546-554, 2011.

[13] M. Hasegawa, H. Hirai, K. Nagano, H. Harada, and K. Aihara, "Optimization for centralized and decentralized cognitive radio networks," Proceedings of the IEEE, vol. 102, no. 4, pp. 574-584, 2014.

[14] Y. Zhao, S. Mao, J. O. Neel, and J. H. Reed, "Performance evaluation of cognitive radios: metrics, utility functions, and methodology," Proceedings of the IEEE, vol. 97, no. 4, pp. 642-659, 2009.

[15] S. M. Zafaruddin, I. Bistritz, A. Leshem, and D. Niyato, "Distributed learning for channel allocation over a shared spectrum," IEEE Journal on Selected Areas in Communications, vol. 37, no. 10, pp. 2337-2349, 2019.

[16] J. Nash, "Non-cooperative games, Second Series," The Annals of Mathematics, vol. 54, no. 2, pp. 286-295, 1951.

[17] K. Akkarajitsakul, E. Hossain, D. Niyato, and D. In Kim, "Game theoretic approaches for multiple access in wireless networks: a survey," IEEE Communications Surveys \& Tutorials, vol. 13, no. 3, pp. 372-395, 2011.

[18] A. Bhattarai, P. Suksompong, C. Charoenlarpnopparut, and P. Komolkiti, "Counter-intuitive channel allocation improvement in distributed cognitive radio networks by adding optimal interference in user's utility," in 2017 14th IEEE Annual Consumer Communications \& Networking Conference (CCNC), pp. 225-230, Las Vegas, NV, USA, January 2017. 
[19] J. Huang, R. A. Berry, and M. L. Honig, "Distributed interference compensation for wireless networks," IEEE Journal on Selected Areas in Communications, vol. 24, no. 5, pp. 1074-1084, 2006.

[20] K. J. Ray Liu and B. Wang, Cognitive Radio Networking and Security, a Game-Theoretic View, Cambridge Books Online, Cambridge University Press, 2012.

[21] A. Bhattarai, P. Komolkiti, and C. Aswakul, "Improved bandwidth allocation in cognitive radio networks based on game theory," in 2013 10th International Conference on Electrical Engineering/Electronics, Computer, Telecommunications and Information Technology, pp. 1-6, Krabi, Thailand, May 2013.

[22] Y.-B. Li, R. Yang, and F. Ye, "Non-cooperative spectrum allocation based on game theory in cognitive radio networks," in 2010 IEEE Fifth International Conference on Bio-Inspired Computing: Theories and Applications (BIC-TA), pp. 11341137, Changsha, China, September 2010.

[23] A. Bhattarai, C. Charoenlarpnopparut, P. Suksompong, and P. Komolkiti, "Developing policies for channel allocation in cognitive radio networks using game theory," in 2014 11th International Conference on Electrical Engineering/Electronics, Computer, Telecommunications and Information Technology (ECTI-CON), pp. 1-6, Nakhon Ratchasima, Thailand, May 2014.

[24] A. Bhattarai, C. Charoenlarpnopparut, P. Suksompong, and P. Komolkiti, "Policies for channel allocation in cognitive radio networks using game theory," ECTI Transactions on Electrical Engineering, Electronics, and Communications, vol. 14, no. 1, pp. 36-48, 2016.

[25] D. P. Agrawal and Q.-A. Zeng, "Introduction to wireless and mobile systems, 3.4 free space propagation," Cengage Learning, 60-65, 2011.

[26] D. B. R. Rawat, "Path Loss," MATLAB central file exchange, 2007, https://www.mathworks.com/matlabcentral/ fileexchange/14016-path-loss.

[27] H. G. Schantz, "A near field propagation law \& a novel fundamental limit to antenna gain versus size," in 2005 IEEE Antennas and Propagation Society International Symposium, vol. 3A, pp. 237-240, Washington, DC, USA, July 2005.

[28] C. E. Shannon, "Communication in the presence of noise," Proceedings of the IRE, vol. 37, no. 1, pp. 10-21, 1949.

[29] A. J. Goldsmith and P. P. Varaiya, "Capacity of fading channels with channel side information," IEEE Transactions on Information Theory, vol. 43, no. 6, pp. 1986-1992, 1997.

[30] R. Faragher and R. Harle, "Location fingerprinting with Bluetooth low energy beacons," IEEE Journal on Selected Areas in Communications, vol. 33, no. 11, pp. 2418-2428, 2015.

[31] M. G. H. Bell, "The use of game theory to measure the vulnerability of stochastic networks," IEEE Transactions on Reliability, vol. 52, no. 1, pp. 63-68, 2003.

[32] N. Nisan, T. Roughgarden, A. Tardos, and V. V. Vazirani, Algorithmic Game Theory, Cambridge University Press, New York, 2007.

[33] P. M. d'Orey, R. Fernandes, and M. Ferreira, "Empirical Evaluation of a dynamic and distributed taxi-sharing system," in 2012 15th International IEEE Conference on Intelligent Transportation Systems, pp. 140-146, Anchorage, AK, USA, September 2010.

[34] G. W. Scully and University of Texas at Dallas, Optimal taxation, economic growth and income inequality, vol. 115, Springer/Kluwer Academic Publishers, 2002.

[35] K. Yamamoto, "A Comprehensive Survey of Potential Game Approaches to Wireless Networks," in IEICE TRANS. COMMU., vol. E98-B, no. 9, 2015. 